\section{Xavier Blanco}

Université Autonome de Barcelone Espagne

(D) https://orcid.org/0000-0001-8210-3668
Linguistique informatique et linguistique diachronique : une alliance nécessaire

Computational linguistics and diachronic linguistics:

a necessary alliance

\begin{abstract}
In this paper, we will try to show how formal linguistics applied to the study of diachrony can be a fundamental asset for research (and consequently also for teaching at a university level) in the field of French language and literature. We will treat successively the analysis of the support verbs (§2), the realization verbs $(\S 3)$, the intensity collocations $(\S 4)$ and the pragmatically restricted clichés (§5). In each section, we will present and discuss numerous examples in medieval French accompanied by their published translations into contemporary French.
\end{abstract}

\title{
Keywords
}

Diachronic linguistics, support verbs, realization verbs, intensifiers, pragmatemes

\section{Introduction}

Dans cet article ${ }^{1}$, nous essaierons de montrer comment la description linguistique formelle peut être un atout important, au point de devenir un préalable presque incontournable, pour un enseignement et une recherche de qualité en langue et littérature françaises.

${ }^{1}$ La recherche qui sous-tend cet article a été financée par le Projet COLINDANTE (Las colocaciones intensivas del francés antiguo y su traducción al francés y al español, Proyecto I+D+i PID2019-104741GB-100). Ministerio de Ciencia e Innovación (Espagne). Je remercie Dolors Català, Gaston Gross et Julio Murillo pour leur relecture attentive de cet article. 
Avec ce texte, nous souhaitons surtout présenter quelques réflexions d'ordre méthodologique pouvant intéresser des jeunes collègues chargés d'activités d'enseignement et de recherche au sein de départements de français en Europe, sachant qu'ils/elles doivent être en mesure de prendre en charge des matières diverses orientées à un public d'étudiants de niveaux fort différents et qu'ils/elles doivent à la fois mener à bien une activité de publication leur permettant de franchir les différents processus d'habilitation existants. Une triple compétence (langagière, linguistique et littéraire) leur est ainsi souvent exigée, ce qui requiert une formation méthodologique, il va sans dire solide, mais, par surcroît, plurivalente.

Nous pensons que la linguistique peut constituer la base d'une telle formation et que, comme elle l'a été sans conteste pendant une grande partie du $\mathrm{XX}^{\mathrm{e}}$ siècle, elle peut continuer à constituer une discipline-paradigme au sein des sciences humaines. Nous tâcherons de montrer, en particulier, comment la linguistique informatique, telle qu'elle a été conçue, développée et transmise pendant des décennies par le prestigieux Laboratoire de Linguistique Informatique (LLI) de l'Université Paris 13, sous la direction de Gaston Gross, constitue un outil actuel et efficace permettant de doter d'une base scientifique robuste l'activité d'enseignement et de recherche. Les jeunes enseignants-chercheurs, qui manquent parfois d'assises méthodologiques solides à conséquence d'un certain relâchement de la formation de base en linguistique descriptive et formelle, ne pourront que profiter d'une telle méthodologie, héritière de la meilleure tradition française et européenne.

Nous considérons, par ailleurs, que la formation d'un linguiste et, de façon générale, d'un spécialiste des langues n'est pas complète si elle n'inclut pas une composante de grammaire historique, de linguistique diachronique et d'analyse des textes médiévaux. Certes, cette composante pourra être plus ou moins importante en fonction de la spécialisation ultérieure de chaque professionnel, mais elle se doit d'exister. Or, nous assistons avec regret et une certaine stupeur à la disparation pure et simple de la formation en diachronie au sein des programmes d'études en langue française dans un grand nombre d'universités européennes. Cette formation est souvent remplacée par des sous-disciplines aux apparences plus alléchantes, mais dont l'impact pour la formation globale n'est pas forcément garanti. Le fait de ne pas doter l'apprenant francisant d'une perspective diachronique ne peut qu'avoir des conséquences négatives ; d'abord, parce qu'il le prive de connaissances qui sont objectivement nécessaires ; ensuite, parce que cela suppose l'interruption d'une tradition qui le coupe d'une très grande partie de la réflexion linguistique de ses prédécesseurs (même quand ils ne travaillaient pas directement sur des sujets de diachronie) ; finalement, parce que cela implique de passer sous silence le rôle central qu'ont joué la langue et la culture françaises au cœur de l'Europe et revient à nier que la transmission d'une langue et d'une culture est toujours liée à la transmission de certaines valeurs² ${ }^{2}$.

${ }^{2}$ Dans les années 90 du siècle dernier, le syntagme dimension européenne était bien présent dans les programmes d'éducation au sein de l'Union européenne. Un effort de consolidation de la 
Il ne faut pas, non plus, oublier que la formation en linguistique historique implique une certaine formalisation. Or, il y a lieu de souligner, telle est notre conviction, que le bannissement de disciplines comme la linguistique historique précède celui de la linguistique générale et que ces deux dérives sont, en grande partie, dues à des raisons de facilité et à une tentative d'élargissement de la base (augmenter le nombre d'étudiants) par la banalisation de la matière enseignée. Cela est d'autant plus inquiétant que ce processus a, assez rapidement, des effets multiplicatifs. Les étudiants n'ayant pas reçu une formation initiale solide en linguistique ne sont plus à même de la dispenser. Il ne s'agit pas du genre de compétence qui puisse s'acquérir par des formations rapides et ponctuelles à caractère complémentaire.

\section{2. À propos des verbes support}

En proposant à nos étudiants la lecture de La Conquête de Constantinople de Geoffroy de Villehardouin ${ }^{3}$ (chronique historique considérée comme le point de départ de la prose française, rédigée vers l'année 1207) et de l'ouvrage homonyme de Robert de Clari (rédigé peu après, vers 1216), nous pouvons leur faire remarquer l'emploi de faire comme verbe support presque universel pour les noms d'action (X. Blanco, 2018). Dans les exemples qui suivent, nous mettons en gras le verbe support en question et nous soulignons le nom prédicatif qu'il prend (du point de vue strictement syntaxique) comme complément d'objet direct :

(1) si mist ses gens en await et fist ses embuskemens (Clari, p. 146)

(2) qui li aidoit a faire toutes ches malaventures (Clari, p. 76)

(3) il ne firent onques traïson (Villeh., p. 150)

(4) Seigneur, nous ferons volentiers markié a vous (Clari, p. 52)

(5) li cuens de Saint Pol fist le jugement que aussi devoit il partir comme uns chevaliers (Clari, p. 192)

(6) les genz li firent la feauté (Villeh., p. 182)

notion d'identité européenne semblait souhaitable et naturel. Il s'agissait de tenir compte de cette entité historique, culturelle et politique de premier ordre (où des notions comme la tolérance, la laïcité et la démocratie avaient trouvé leur berceau) et de la mettre en valeur, sans pour autant nier ses aspects moins brillants.

${ }^{3}$ Nous indiquons les sources de nos exemples en bibliographie, sauf pour les exemples qui ont été extraits des bases textuelles (Frantext ou BFM, Base de Français Médiéval) ou de dictionnaires. Pour les sources secondaires consultées, voir également la bibliographie en fin d'article. 
Voici comment l'éminent romaniste Jean Dufournet a traduit ces énoncés vers le français contemporain :

(1') il mit ses soldats aux aguets et dressa son embuscade

(2') qui l'aidait à perpétrer tous ses forfaits

(3') ils ne commirent jamais de trahison

(4') Seigneurs, nous conclurons volontiers un marché avec vous

(5') le comte de Saint-Pol rendit ce jugement : il devait participer au partage tout comme un chevalier

(6') les gens lui jurèrent fidélité à l'empereur

Nous pouvons relever comment la traduction exige, soit pour des raisons stylistiques, soit pour des raisons purement grammaticales, l'introduction d'une variété de formes correspondant à des verbes support dont l'ancien français ne se servait pas. L'exemple suivant montre comment l'ancienne langue ne craignait pas la répétition d'un support donné, ce que le français contemporain ne tolère pas facilement :

(7) fist tant de si grans desloiautés, que onques nus traïtres ne nus mourdrissierres tant n'en fist comme il fist (Clari, p. 74)

(7') il commit plus de félonies qu'aucun traître qu'aucun meurtrier en fit jamais

Les cas de ce type de répétition abondent en ancien français. Dans (7'), le traducteur a recours, d'une part, à la mise en facteur commun et, d'autre part, au verbe support approprié aux $<$ délits $>^{4}$. En ancien français, des substantifs comme murtres, outrage, péchié, traïson, vilenie sélectionnaient ${ }^{5}$ le verbe support faire. La forme commettre ne commencera à être utilisée comme support approprié

${ }^{4}$ L'étiquette < délits> correspond à une classe d'objets (D. Le Pesant, M. Mathieu-Colas, 1998) d'actions qui ont comme opérateurs appropriés commettre, se rendre coupable de, condamner qqn pour, etc.

${ }^{5}$ Rappelons ici qu'un verbe support régit son nom prédicatif du point de vue syntaxique (en général, en position d'objet direct, plus rarement en position de sujet - verbes support d'occurrence - et, plus rarement encore, dans le cas du français, en position d'objet indirect). Par contre, du point de vue lexical, c'est le verbe support qui est sélectionné par le nom prédicatif. Si la sélection d'un certain support peut être observée par rapport à tous les éléments d'une classe d'objets (ce qui est souvent le cas), on peut parler de sélection sémantique. La sélection sémantique peut être le résultat d'une extension analogique d'une première sélection de type lexical. Les verbes support qui présentent une distribution large mais uniforme ( $\mathrm{p}$. ex. perpétrer $<$ délits-crimes $>$ ) relèveraient d'une sélection sémantique puisqu'un néologisme correspondant sémantiquement à un <délit-crime $>$ se combinerait, en toute probabilité, avec le verbe support perpétrer. Précisons, toutefois, que la langue est en évolution constante et qu'il faut également tenir compte des phénomènes de discours (extensions de sens, emplois métaphoriques, jeux verbaux, etc.). 
pour $<$ délit $>$ qu'à partir du $\mathrm{XIV}^{\mathrm{e}}$ siècle $^{6}$. Cf. les exemples suivants (s.v. commettre du $\mathrm{DMF})^{7}$ :

(8) Item, aucune fois un homme commet et fait adultere tant seulement pour cause et afin de gaaignier et de prendre aucun proffit. (E. A. ORESME, c. 1370,280$)$

(9) Et plus ne scet ne n'est record d'autres larrecins ou mauvaistiez par lui faites et commises, si comme il a affermé par serement. (Reg. crim. Chât., I, 13891392, 135)

Ce qui vient d'être signalé n'implique pas que les verbes support appropriés à certaines classes de prédicats n'existaient pas en ancien français. Il semblerait, cependant, qu'ils conservaient une partie de leur sémantisme original. Voici quelques exemples tirés des mêmes ouvrages que ceux présentés plus haut :

(10) si canta on une messe (Clari, p. 186)

(10') on chanta une messe

(11) Quant la messe fu dite (Villeh., p. 26)

(11') Quand la messe fut dite

La messe peut être dite ou chantée. D’un côté, ce verbe a le fonctionnement d'un support. Il actualise le prédicat nominal messe en temps, mode et aspect. Il peut fonctionner comme synonyme discursif de célébrer. Mais il conserve un côté prédicatif, car il existe bel et bien une différence entre une messe dite et une messe chantée, même si progressivement chanter la messe s'est employé de plus en plus comme synonyme de dire la messe et s'est également appliqué, en conséquence, aux messes lues. Autrement dit, le verbe s'est progressivement désémantisé pour devenir, dans certains cas, un support pur.

Considérons un autre exemple, cette fois avec un nom prédicatif d'état :

(12) avoit langui tot l'iver d'une fièvre quartaine (Villeh., p. 169)

(12') avait langui tout l'hiver d'une fièvre quarte

${ }^{6}$ C'est le cas, également, pour perpétrer : Et dit que ce sont tous les crimes et deliz qu'il a faiz, commis et perpetrez (Reg. crim. Chât., II, 1389-1392, 70) (cf. DMF s.v. délit).

${ }^{7}$ Une situation tout à fait comparable peut être observée à propos de l'espagnol cometer (R. García Pérez, 2005 : 325). Il est important de signaler qu'un des intérêts de l'étude de la langue médiévale (et non des moindres) est de mettre en évidence la parenté intime des langues romanes et, jusqu'à un certain point, du Standard Average European (SAE) qui relève davantage d'une communauté historico-culturelle que d'une communauté strictement linguistique. 
Le verbe languir a bien son sens prédicatif (que nous pourrions paraphraser par 'demeurer diminué par une maladie ou par les symptômes de celle-ci'). L'armée croisée a été privée du secours d'un de ses chefs militaires qui, alité, n'a pas pu porter les armes pendant une longue période. On y perçoit le latin languor 'faiblesse, abattement'. En même temps, languir fonctionne aussi comme support approprié de fièvre quarte (nom assimilé à un nom de <maladie>) et il serait tout aussi possible d'écrire : il avait souffert d'une fièvre quarte ou même il avait eu la fièvre quarte.

Le sens « initial» d'un support reste toujours accessible et peut engendrer des jeux verbaux : Ainsi par exemple, le verbe support souffrir est resémantisé dans des énoncés comme : Je ne souffre pas (de démence, d'une déviation sexuelle), j'en jouis!

Au-delà de l'ancien français et du moyen français, l'alexandrin classique n'a certainement pas été étranger au développement des verbes support en français, car son rythme favorise l'utilisation de structures à verbes support et la rime profite de la récurrence des suffixes des noms prédicatifs. Certes, nous ne sommes absolument pas en mesure de retracer ici, ne serait-ce qu'en ébauche, une histoire des verbes support. Toutefois, nous relèverons deux commentaires de Voltaire sur l'utilisation des verbes support de la part de Corneille pour montrer jusqu'à quel point la sensibilité linguistique a pu évoluer assez rapidement concernant cet usage. Voltaire affirme, à propos du vers suivant d'Héraclius (pièce de 1647) : Quel dessein faisiez-vous sur cet aveugle inceste?

On ne fait point l'estime : cela n'a jamais été français ; on a de l'estime, on conçoit de l'estime, on sent de l'estime; et c'est précisément parce qu'on la sent qu'on ne la fait pas. Par la même raison on sent de l'amour, de l'amitié ; on ne fait ni de l'amour, ni de l'amitié.

(Voltaire, 1797, t. 8, p. 142)

Et, il s'exclame, par rapport au vers suivant de Nicomède (pièce datant de 1651): Vous offenseriez l'estime qu'elle en fait :

Cela n'est pas français ; On ne peut dire : faire un dessein ; on dit bien : concevoir, former un dessein, mon dessein est d'aller, j'ai le dessein d'aller, etc.

(Voltaire, 1797, t. 7, p. 170)

Indépendamment de leur pertinence du point de vue de la critique littéraire, ces commentaires sont précieux pour l'historien de la langue dans la mesure où ils révèlent quelle était la perception de ces séquences à verbe support plus nom prédicatif écrites vers le milieu du XVII ${ }^{e}$ siècle par un auteur écrivant aux alentours de 1764 (date de la première édition des Commentaires sur Corneille). Rappelons que Voltaire est né dix ans après la mort de Corneille. La distance temporelle 
entre les deux grands hommes n'est donc pas aussi considérable que l'on pourrait penser au vu des dates qui viennent d'être rappelées.

\section{3. À propos des verbes de réalisation}

Nous avons parlé ci-dessus des verbes support, dont le sens lexical s'est progressivement estompé pour leur permettre de devenir en priorité justement des supports grammaticaux des noms prédicatifs; il existe un autre type de verbes qui, tout en présentant la caractéristique fondamentale des collocatifs, c'est-à-dire le fait d'être sélectionnés en principe de façon lexicale (sélection généralisable à certaines classes sémantiques) conservent un sens général que nous pourrions paraphraser comme 'réaliser les objectifs inhérents à la dénotation de la base nominale'. Il s'agit des verbes de réalisation ${ }^{8}$. Quelques exemples en français contemporain pourraient être : fumer un cigare, lire un roman ou visiter un musée. Pustejovsky (2016) se sert du terme rôle télique pour désigner des combinaisons comme to read a novel ou to drive a car.

Il y a lieu de remarquer que le fait de constituer une liste de verbes de réalisation constitue une façon particulièrement efficace et relativement simple de relever, par un moyen strictement linguistique, et donc moins dépendant de la subjectivité du chercheur, les notions-clé d'un texte donné (pourvu que celui-ci soit d'une certaine extension, p. ex. un roman, une pièce de théâtre) et de les situer dans le contexte historique auquel ils appartiennent. Ainsi, par exemple, un verbe de réalisation tout à fait courant pour les $<$ textes $>$ au Moyen Âge est ouïr ('entendre, écouter') dans la mesure où les textes étaient lus à voix haute à l'intention de leurs destinataires (quand il s'agissait de lettres ou de messages, cf. ci-dessous §5) ou, plus largement, à l'intention du public (dans le cas de textes littéraires).

Nous avons relevé tous les verbes de réalisation présents dans la chanson de geste anglo-normande de la fin du XII ${ }^{\mathrm{e}}$ siècle Beuve de Hamptone ${ }^{9}$. Cette opération nous a permis, d'une part, de mettre en évidence toutes les classes syntactico-semántiques, ou classes d'objets, importantes pour le texte en question. Ainsi, par exemple, par rapport à espeie, brant ('épée') on a une réitération de verbes comme ceindre ('ceindre'), sacher, traire ('dégainer'), avaler, ferir de ('enfoncer, frapper avec') qui correspondent aux trois degrés de réalisation d'un substantif de type <arme blanche $>$ : 'porter'10, 'dégainer' et 'frapper'. Ces verbes constituent

\footnotetext{
${ }^{8}$ Pour ce terme-notion, cf. Mel'čuk et Polguère (sous presse).

${ }^{9}$ Pour une présentation beaucoup plus détaillée des résultats, cf. X. Blanco (2020).

${ }^{10}$ Nous faisons référence à une acception technique de porter, qui est nominalisable : le port d'une arme, qui ne se confond pas avec le transport d'une arme. Le sens de ceindre en ancien français peut correspondre à 'porter' ou à son inchoatif.
} 
le noyau des « opérateurs appropriés » (G. Gross, 2012) pour une classe d'objets (la notion d'opérateur approprié est pourtant plus large que celle de verbe de réalisation).

Il est crucial de tenir compte de ces prédicats, en particulier quand on étudie une langue à la graphie peu fixée et que l'on ne dispose pas de l'intuition linguistique d'un locuteur natif. En effet, le fait de ne pas être attentif à la combinatoire lexicale restreinte d'un substantif donné peut donner lieu à des erreurs de traduction graves. En voici un exemple : la traduction à l'anglais (Boeve de Haumtone, trad. de J. Weiss, 2008) de la chanson de geste en question, globalement excellente, contient cependant une erreur réitérée. Le vers 139 (Un escu a son col, en sa mein un espé) est traduit par : with a shield round his neck and a sword in his hand. Or, il ne s'agit pas d'une épée (sword), mais d'un épieu (une sorte de lance conçue surtout pour la chasse, mais dont on se servait aussi pour la guerre). On ne voit pas, en effet, pourquoi un chevalier partirait en voyage (à cheval) l'épée à la main. Il est, par contre, normal qu'il voyage une arme d'hast à la main. La forme espé est ambiguë. Judith Weiss l'interprète de façon incorrecte aux vers 139, 547, 2607 et 2931, mais elle ne se trompe pas quand un opérateur approprié (en l'occurrence, le méronyme 'hampe') lui fait comprendre qu'il s'agit nécessairement d'une arme d'hast : (3494 E brise la hante de l'espé trenchant : broke Fabur's lance with his sharp blade). Dans ce cas, elle traduit par lance.

Considérons un autre exemple. Les messagers et les hérauts (figures de grande importance dans l'univers de la chanson de geste) présentent le verbe de réalisation mander ('envoyer un messager à qqn') (traduction de Robert Martin) :

(13) Mesagers manderum par ample païs ! (v. 3051)

(13') Nous allons envoyer des messagers à travers de vastes pays!

(14) Un messager unt al roi envëez (v. 3067)

(14') Un messager a été envoyé au roi Hermin

Il ne faut pourtant pas confondre ce verbe de réalisation avec un autre emploi de mander qui correspond à 'faire venir quelqu'un, lui donner avis ou ordre de venir' (souvent par référence à un domestique, mais qui peut s'appliquer à n'importe quel subordonné, y compris à un messager, à quelqu'un ayant une fonction sociale ou une profession dont on a besoin ou, de façon encore plus générale, à quelqu'un avec qui on souhaite s'entretenir à propos d'un quelconque sujet). C'est le cas dans le vers 3522 de Beuve de Hamptone, où le roi fait venir un messager pour lui transmettre le message qu'il devra porter. Il serait donc erroné de traduire par Le roi a envoyé un messager, car il s'agit bien du contraire (même si c'est exprimé par une même forme, mander) : 
(15) Le roi ad un mesager mandé / Vos en irez a Civile la cité / [...] (v. 3522-3523) (15') Le roi a fait venir un messager / Vous allez vous rendre à Civile / [...]

Prenons un dernier exemple. Les substantifs désignant des hommes d'Église (prestre, eveske) présentent un certain nombre d'opérateurs appropriés. La plupart sont facilement identifiables en tant que tels. Mais le vers suivant peut poser problème :

(16) Mande l'eveske, si se est conseylé (v. 3330)

Même si les dictionnaires d'ancien et de moyen français (comme le GD, l'AND ou le DMF, cf. Références) ne contemplent pas cette acception, le contexte élargi met bien en évidence que le sens de conseiller est ici 'se confesser'" : Le roi se fist a muster porter / Mande l'eveske, si se est conseylé / E de tuz sé pechez deliverez / A Dampnedeu est il ben acordé (v. 3329-3332).

Nous pouvons observer que le travail sur les verbes de réalisation et, de façon plus large, sur les opérateurs appropriés à une classe syntactico-sémantique nous dote d'une assise solide pour l'étude non seulement linguistique, mais aussi historique et littéraire d'un texte. La linguistique (et non seulement la philologie) peut ainsi s'avérer une science auxiliaire ${ }^{12}$ de premier ordre pour l'historien.

Une science est d'autant plus nécessaire qu'elle peut être mise au service d'un plus grand nombre de disciplines. C'est, sans aucun doute, le cas de la linguistique, qu'elle soit synchronique ou diachronique. Soulignons à ce propos que nous plaidons ici la cause de la linguistique autant que celle de la philologie. La linguistique diachronique est, certes, étroitement imbriquée avec la philologie, mais elle s'en distingue par sa vocation à rendre compte du fonctionnement de la langue comme système, alors que la philologie a une forte spécialisation dans les textes écrits ${ }^{13}$ et, donc, un penchant beaucoup plus net pour l'étude du discours que pour la reconstruction du système de la langue. Or, c'est précisément la comparaison scientifique entre la langue (au sens saussurien du terme) d'une certaine époque et la langue de nos jours qui peut nous prémunir contre le plus grand risque qui guette toute étude historique : l'anachronisme. En effet, seulement la description systématique d'un état de langue révolu peut se substituer au manque d'intuition linguistique du locuteur natif ${ }^{14}$, notion-clé en linguistique mais aussi en stylistique

${ }^{11}$ Le vers est, par ailleurs, traduit : il fit venir l'évêque, se confessa à lui.

${ }^{12}$ Les sciences auxiliaires de l'histoire (archéologie, épigraphie, généalogie...) sont dites aussi « sciences ancillaires », ce qui, à notre avis, n'est nullement péjoratif, car... quelle serait la vocation d'une science sinon de servir, de se rendre utile?

${ }^{13}$ Le plus souvent littéraires, mais non de façon exclusive.

${ }^{14}$ Nous avons quand même à notre disposition les commentaires métalinguistiques qui nous sont restés, qu'ils proviennent de sources lexicographiques ou de jugements stylistiques produits à propos de certains énoncés (cf. les propos de Voltaire que nous avons recueillis ci-dessus). 
comparée. Toute stylistique se base, d'une façon ou d'une autre, sur l'évaluation d'une série d'écarts dans le maniement de la langue qui se manifestent dans le discours. Toute stylistique est, donc, une stylistique différentielle. Pour l'interprétation des textes du passé (et, bien entendu, pour l'appréciation des textes littéraires), les états de langue passés sont à reconstruire, tâche qui est spécifiquement une tâche linguistique.

\section{4. À propos des intensifs}

Parmi les collocations, celles qui expriment l'intensité sont les plus nombreuses, aussi bien en langue qu'en discours. Elles sont très variables en fonction des époques, des genres textuels et des niveaux de langue et elles peuvent colorer fortement un texte. Même si leur sens de base est l'intensité, l'expression de ce sens n'épuise pas leurs valeurs connotatives, expressives et évaluatives.

Il est essentiel de bien reconnaître et décrire l'expression lexicale de l'intensité présente dans un texte donné afin d'éviter des erreurs d'interprétation (et a fortiori de traduction) graves et de le situer au sein d'une tradition linguistique et littéraire.

Comme pour les verbes support, nous constatons d'abord une présence faible de collocatifs intensifs en ancien et moyen français par rapport au français contemporain. Observons les exemples suivants, toujours extraits de Beuve de Hamptone :

(17) Puis avint cel jur que mult eniré fu

(17') mais vint un jour où il le regretta amèrement (v. 22)

(18) Mult en fust corucé Boefs de Hamtone

(18') Et Beuve de Hamptone, vivement irrité (v. 1199)

(19) Mes ne lessent, mult l'unt fet blescer

(19') Ils ne la lâchent pourtant pas, et la blessent sévèrement (v. 1669)

(20) "Sire, mult sui malades, ne dorrai longement"

(20') "Sire, je suis très gravement malade, il ne me reste guère à vivre » (v. 3811)

Nous avons sélectionné des exemples où l'adverbe mult a été traduit par des collocatifs adverbiaux en -ment. Il serait banal d'observer, en effet, que ces combinaisons verbe-adverbe en -ment ne sont pas librement interchangeables (?regretter gravement, ??vivement malade) même si une certaine variation dans la sélection 
de l'adverbe reste possible. D'ailleurs, il serait aisé de donner un grand nombre d'autres exemples ou un adverbe à très large spectre combinatoire de l'ancien français est traduit par des collocatifs. Toujours dans l'ouvrage cité, nous trouvons : Grant coupe = coup violent $($ v. 3250$) ;$ penance grant $=$ sévère pénitence (v. 3380) ; Grant ert la perte = les pertes seront lourdes (v. 3572), etc.

Cette constatation ne veut pas dire que les collocatifs intensifs sont inconnus au Moyen Âge. En effet, nous trouvons des cas comme (Lancelot du Lac : Le Val des Amants infidèles, début du XIII ${ }^{\mathrm{e}}$ siècle, éd. Yvan G. Lepage) :

(21) Ha! Dieus, tant rechevra hui laide pierte la riche Table Ronde (p. 126)

(22) anchois a tant alé a finne forche ke devant la porte est venus (p. 234)

(23) pour plus pesant colp douner (p. 284)

(24) dont la noire doleurs li prist au ceur, ki onques nel laissa (p. 396)

Il est essentiel de connaître ces collocations intensives afin de pouvoir évaluer un texte. En effet, comme il a déjà été signalé, nous ne pouvons apprécier un écart que si nous connaissons la situation non marquée. La stylistique médiévale a besoin d'une description systématique des collocations intensives dont elle ne dispose pas jusqu'à présent.

Il ne nous est évidemment pas possible de rendre compte ici de l'ensemble de ces collocations. Nous ferons donc quelques observations ponctuelles référées à un type de collocation précis : les comparatives intensives prenant comme deuxième terme de la comparaison un nom de couleur. Il s'agit de séquences courantes en français médiéval dont le sens littéral est : 'plus blanc que la neige sur l'arbre', 'plus noir que l'aile d'un corbeau', 'plus rouge que le sang', 'plus vert qu'une feuille de lierre', 'plus jaune que la cire', etc.

Nous avons repéré 600 contextes de ce type pour le blanc, 300 pour le noir, 200 pour le rouge, 50 pour le vert et 50 aussi pour le jaune dans des textes littéraires entre le $\mathrm{XII}^{\mathrm{e}}$ et le $\mathrm{XV}^{\mathrm{e}}$ siècle (X. Blanco, sous presse). Ce n'est qu'après avoir réuni, formaté et classé une quantité consistante d'exemples en suivant une méthodologie propre à la lexicographie informatique que l'on peut commencer à mettre en évidence certaines régularités (et, donc, certaines déviations) et à faire des observations qui ne sont pas possibles (ou à peine) si l'on raisonne sur un nombre d'unités restreint et sans disposer de données formalisées.

La couleur noire présente plusieurs parangons dans la langue médiévale, certains le sont encore de nos jours (le charbon, la mûre, le corbeau), d'autres nous résultent plus étranges en tant que représentants du noir (la terre, le fer, la poi$\left.v_{\text {vré }}{ }^{15}\right)$. Une des comparaisons figées les plus fréquentes est noir com arrement

${ }^{15}$ La terre est symboliquement noire au Moyen âge, tout comme l'eau est verte ou l'air est blanc. Précisons que la désignation de la couleur est, en grande mesure, un phénomène social et culturel. Le fer peut être jugé noir par référence au sulfate de fer (le vitriol), employé comme pigment. Le fait que fer rime avec enfer favorise grandement l'usage de comparaisons axées sur ce terme dans certains 
ou plus noir qu'arrement (triblé, destrempé). Arrement désigne la matière que l'on fait fondre pour obtenir de l'encre. Nous observons que les comparaisons du type 'noir comme de l'encre' (suite parfaitement possible de nos jours) sont tardives ${ }^{16}$. Les comparaisons basées sur arrement sont, par contre, plus anciennes. Nous en trouvons déjà une occurrence dans La chanson de Roland (Ki plus sunt neirs que nen est arrement, Frantext E174, 1125, p. 150) et jusqu'à cinq cas dans Aliscans (fin du XII ${ }^{\mathrm{e}}$ siècle) (éd. Wienbeck, Hartnacke) :

(25) Noire ot la char plus qu'arrement triblés (v. 44)

(26) Morgans li faés / Ki plus est noirs ke aremens triblés (v. 4395)

(27) N'ot pas destrier, ains cevauce jument... / Et l'uns et l'autre est noirs com arrement (v. 5712-5715)

(28) Noirs est li rois com arrement triblés (v. 6672)

(29) Les caveus noirs com arrements triblés (v. 7262)

Or, il faut tenir compte du fait qu'encre provient du bas latin encau(s)tum « encre de pourpre (réservée à l'empereur)» (cf. TLFi). À l'origine, encre désigne donc une substance rouge. Tandis que arrement provient du latin atramentum, dérive de ater 'noir mat'. Le latin niger 'noir brillant' a donné différentes formes qui sont encore d'usage (noir, noircir..., dénigrer). Ater, par contre, ne subsiste que très indirectement dans atroce (atrox 'à l'aspect noir', d'où 'affreux, terrible'). La forme arrement disparaît au XVI siècle, coïncidant avec la généralisation de la composition pâteuse appelée encre pour l'impression des caractères typographiques en imprimerie ${ }^{17}$.

Concernant le caractère figé des comparaisons qui nous occupent, il est intéressant d'observer que, comme le signale Charles Potvin dans son édition du Conte du Graal (1866-71), le terme arrement n'était probablement plus compris par le traducteur de 1530. En effet, ce traducteur anonyme rend la séquence plus noire d'airement par merveilleusement noire, ce qui nous confirme qu'il percevait l'expression comme une indication d'intensité (avec, peut-être, un certain caractère mélioratif). Notons qu'un locuteur peut très bien saisir le sens collocationnel d'une expression intensive sans pour autant être capable d'en reconstituer le sens littéral (une peur bleue, fier comme un pou... sont des collocations que les locuteurs comprennent, mais qu'ils ont bien de mal à motiver).

contextes. Quant à poivrée (pevree en ancien français), il s'agit d'une sauce au poivre ou de certains plats basés sur une sauce au poivre.

${ }^{16} \mathrm{P}$. ex. Item, en la playne d'icelle montaigne et souffrerie a deux sources de eaue dont l'une est chaulde et noire comme encre (Frantext 0702 : André de la Vigne, Le voyage de Naples, 1495, p. 264).

${ }^{17}$ Le rapport entre cette comparaison figée et l'imprimerie est fort plausible. Il serait à noter, par exemple, qu'il n'y a pas d'exemples en espagnol de negro como la tinta antérieurs au XVI' siècle. 
Passons au vert. Au Moyen Âge, le vert est associé à la perte du teint du visage, ce qui n'est pas évident pour un lecteur contemporain. Il s'agit d'un vert cadavérique. En effet, un certain vert non saturé, plutôt un verdâtre (subviridis en latin médiéval), est la couleur de la maladie, de la chair décomposée, des cadavres et des revenants. Nous sommes habitués aux lividités cadavériques (livor mortis), qui sont plutôt bleuâtres ou violacées et qui se forment en quelques heures. Mais l'homme médiéval devait bien connaître aussi les tâches vertes qui se forment sur la paroi de l'abdomen provenant de la migration des matières fécales, vision que la thanatopraxie moderne nous épargne. Par ailleurs, la disparition massive des globules rouges lors du décès provoque un ictère (jaunisse) qui est décrit comme vert, souvent à l'aide de comparaisons figées intensives.

Dans le Roman de Troie (éd. Joly, v. 21570-21572) un combattant blessé qui a perdu une grande quantité de sang est à l'agonie : Li sans li cort jusqu'al talon / Parmi les mailles del blazon / Tant en a perdu que sovent [...]. Il est décrit comme étant : Plus verz, plus pale que n'est cendre (v. 21574). Alexandre, empoisonné, devient plus vert qu'un poireau : Quant li reis ot beü, si li fredi lo cors, / Sa carn devint plus verz que n'est foille de pors (Roman d'Alexandre, éd. E. C. Armstrong et al., v. 171-172).

Villon se sert d'une comparaison similaire dans son Testament (éd. Thiry) :

(30) En sang c'on voit es poillectes sechier

Sur ces barbiers, quant plaine lune arrive,

Dont l'un est noir, l'autre plus vert que cyve (Testament, 1444-1446)

Les poillectes ou pallectes sont les écuelles où les barbiers recueillaient le sang des saignées. Dans ce sang, prétend Villon, il faudrait faire frire les langues ennuyeuses. Du sang qui présente le vert de la putréfaction ('plus vert qu'une ciboule').

Il n'y a pas de doute que ce vert serait perçu par nos contemporains (et par nous-mêmes) comme jaunâtre. Il n'est pas étonnant que les dénominations associées au vert et au jaune aient connu des fluctuations et des hésitations (le latin galbus, étymon de jaune, fait référence à une couleur jaune-verte ; la forme russe зелёный 'vert' est apparentée à jaune et à yellow). Il faut aussi tenir compte du fait qu'un grand nombre de plantes, parangon par excellence du vert, deviennent jaunes à certaines époques (pensons aux céréales) : (jaune, blond) comme les blés n'a de sens que si l'on pense aux blés mûrs ; et nous trouvons aussi des exemples en français contemporain de vert comme les blés.

Terminons en mentionnant une particularité des comparaisons figées faisant référence à la couleur jaune. Alors que des formes comme blanc, noir, rouge ou vert (et leurs variantes) se combinent avec un certain nombre de deuxièmes termes de la comparaison, la dénomination de la couleur jaune varie beaucoup en fonction dudit deuxième terme. Ainsi, on est blond comme l'or, mais jaune 
comme cire et roux comme (le jaune d') euf. Les cheveux qui seraient blonds (ou sors $^{18}$ ) comme l'or ${ }^{19}$ est l'une des comparaisons (très positivement connotée) les plus répandues au Moyen Âge. On la retrouve dans le nom de deux des héroïnes les plus célèbres de la littérature médiévale : Iseut la Blonde et Soredamor (tout comme on reconnait la comparaison 'blanche comme fleur de lys' dans Blanchefleur). Le collocatif cire, quant à lui, est à comprendre comme 'cire d'abeille' (il ne s'agit pas de la cire à cacheter des lettres, associée au rouge). Cette comparaison se réfère à un symptôme de dérèglement physique ou émotionnel. Dans cette comparaison, la couleur jaune est prise en mauvaise part. Aliste (la fausse Berthe dans Berte aus grans piés d'Adenet le roi) dit :

(31) - Mere, ce dist la serve, je suefre tel martire / Que j'en suis aussi jaune devenue com cire. (v. 2116-2117)

La forme ros ('qui tire sur le jaune orange, roux', cf. DMF) désigne différentes nuances du jaune. Elle a, dans ces comparaisons, le sens 'blond' (non pas de 'roux') :

(32) [...] il estoit bien forny et hault de tous ses membres et sa cher estoit blanche comme lis, et les yeulx vers et amoureux; ses cheveux roux comme fin or. (C. Belle Maguel, 1453 : 26) (DMF, s.v. roux)

Remarquons à cet égard que le catalan ros - qui provient aussi du latin russus - s'applique aux cheveux et est l'équivalent courant de blond.

La comparaison 'roux comme (le jaune d')œuf' ${ }^{20}$ prend toujours 'dents' comme premier terme de la comparaison. L'exemple de départ est celui qui se trouve dans l'antiportrait de la Demoiselle hideuse du Conte du Graal (éd. Ch. Méla) :

(33) Ses danz resanble[nt] moiel d'euf / De color, tant estoient ros (v. 45594560)

Elle a été empruntée par d'autres auteurs, comme dans les vers suivants, référés à un nain dans The Continuations of the Old French Perceval of Chrétien de Troyes (éds. W. Roach, R. H. Ivy, vol. 2) :

(34) Les danz avoit et granz et lonc, / Einsint jaunes com moiauf d'uef (v. 25522553)

${ }^{18}$ D'une couleur 'jaune-brun' : si cheveus ressambloient fil d'or / Et n'estoient ne trop blont ne trop sor (MACH., J. R. Beh., c. 1340, 68, DMF, s.v. sor).

${ }^{19}$ Pour une étude approfondie, cf. M. Rolland-Perrin (2010).

20 'Jaune d'œuf' est dit moiel. 


\section{5. À propos des clichés pragmatiquement restreints}

Le figement ne concerne pas uniquement le niveau de l'unité lexicale, mais il se trouve également de façon massive au niveau morphologique (p. ex. une manchette n'est pas une 'petite manche' mais un ornement de dentelle, etc. s'ajustant aux poignets d'une chemise, entre d'autres acceptions) et au niveau de l'énoncé ${ }^{2}$. Gaétane Dostie (2019) distingue jusqu’à sept types majeurs de «phrases préfabriquées », dont les pragmatèmes.

Dans ce qui suit, nous examinerons quelques clichés pragmatiquement restreints (pragmatèmes) (M. Mel'čuk, 2020) du moyen français. Il s'agit d'unités linguistiques de type clausatif dont l'emploi est lié à certaines conditions extralinguistiques (que ce soient d'ordre temporel, local, de type spécifique de support matériel, etc.) (X. Blanco, S. Mejri, 2018).

Les pragmatèmes étant liés à des situations de communication très précises, les textes médiévaux que nous conservons ne contiennent, sans doute, qu'une petite partie de ceux qui étaient effectivement utilisés. Or, nous avons un type spécial de texte qui est particulièrement riche en pragmatèmes : les Manières de langage. Il s'agit d'une collection de dialogues-modèles rédigés en Angleterre (fin du XIV et début du XV $\mathrm{XV}^{\mathrm{e}}$ siècle $^{22}$ ) qui constituaient des textes d'apprentissage de la langue française à l'usage des anglophones. Ils étaient orientés, en particulier, au voyageur et lui proposaient des phrases et des petits dialogues devant lui permettre de faire face à toute une série de situations quotidiennes : aller chez le boulanger, chez le drapier, saluer, demander des nouvelles, demander l'heure et le chemin, demander une chambre à l'auberge et même requérir d'amour la femme de l'aubergiste!

Nous avons traité de ces ouvrages dans Blanco (2015). Nous voudrions donner ici uniquement trois exemples qui nous semblent pertinents pour illustrer nos propos.

Commençons par signaler, en rapport avec ce qui a été observé dans la section $\S 3$, que certaines classes d'objets peuvent présenter soit des opérateurs appropriés différents à ceux qui sont courants aujourd'hui, soit, du moins, des fréquences très différentes pour certains opérateurs. Il n'est pas du tout exclu, de nos jours, de se faire lire une lettre à voix haute, mais, ce n'est pas la situation la plus courante. La lettre médiévale, par contre, comportait souvent le pragmatème suivant :

${ }^{21}$ Il s'étend même jusqu'à certains ensembles d'énoncés : répliques codées à certains pragmatèmes (De rien) ou textes courts, p. ex. une prière codifiée.

${ }^{22}$ En effet, au moment où les archers anglais et gallois mettaient en pièces la chevalerie française à Azincourt, les ouvrages orientés à l'apprentissage du français étaient très en vogue en Angleterre (en fait, la Manière de langage de 1415 nous donne même une brève description de la célèbre bataille, insérée dans un dialogue didactique). 
(35) A toutz yceux qui cestes letres verront ou orront, Johan d'Orlians de Parys, salutz.

Ou encore :

(36) Sachent toutz gentz qi cestes lettres verront ou orront...

Le verbe oür est, donc, à tous les effets, un prédicat approprié des <textes> médiévaux, comme le montrent aussi les très fréquents incipits (d'ouvrage ou d'épisode) des romans, des hagiographies, des collections de miracles, des nouvelles, etc. où le destinataire est identifié non pas comme lecteur mais comme auditeur $^{23}$ (nous donnons des exemples allant du XII au XV siècle. Dans Conception Nostre Dame de Wace (éd. F. Laurent et al.) (milieu du XII siècle) :

(37) El nom Dé, qui nos doignt sa grace, / Oéz que nos dist maistre Gace (v. 1-2)

Dans le Roman de Tristan (1170) (Béroul, Frantext S708), nous avons :

(38) Oez, seignors, quel aventure : / Tant lor dut estre pesme et dure! (p. 57)

(39) Oez, seignors, quel aventure! / L'endemain fu la nuit oscure (p. 133)

Dans les Cent nouvelles nouvelles (c. 1456-1467) (Frantext 5701) :

(40) enferma son mary ou coulombier par la maniere que vous orrez. (p. 18).

(41) il fut puny en la façon que vous orrez (p. 402).

Et nous trouvons des exemples semblables aussi dans des textes non littéraires, comme des Bestiaires ou des Computs (Philippe de Thaon, Bestiaire, début du XII siècle dans BFM, c. 417—433) :

(42) Pur ço est reis leüns ; / Or oëz ses façuns (v. 29-30)

$\mathrm{Si}$, de nos jours, nous avons des textes oralisés, le Moyen Âge présentait donc, surtout, un oral scripturalisé.

Notons aussi que les salutations correspondant aux différents moments de la journée se distribuaient autrement que de nos jours. On disposait, d'abord, d'une formule (qui pouvait adopter plusieurs variantes) réservée au lever du jour, à la

${ }^{23}$ C'est bien entendu également le cas des chansons de geste (p. ex. les deux premiers vers du Couronnement de Louis : Oiez, seignor, que Deus vos seit aidanz! / Plaist vos oir d'une estoire vaillant, Frantext E173, p. 1), mais, dans ce cas, on fait référence plutôt à la récitation ou au chant qu'à la lecture à voix haute. 
première salutation de la journée (dans Manière de langage 1399, Frantex 7009, p. 29) :

(43) - Sire, boun matyn. I - Sire, boun matyn a vous. I - Bon matyn vous doigne Dieu. I - Sire, bon matin puissez vous avoir. I - Dieu vous donne bon matyn et bon aventure.

Ou encore : Bon matin (et bon encontre, et bon estrayne, et bonne joie, et bonne santee). Ces formules étaient accompagnées de la remarque métalinguistique suivante : Quant vous encontrez ascuny a l'ajournant, vous dirrez ainsi.

Ensuite, nous avons les pragmatèmes suivants toujours accompagnés des indications métalinguistiques qui en précisent les conditions d'emploi (toujours dans ML 1399, p. 29-30) :

(44) A mydy, vous parlerez ainsi :

- Dieu vous donne bon jour et bons heurez. / - Bon jour vous doigne Dieu et bon detinee.

(45) Aprés manger, vous dirrez ainsi :

- Dieu vous donne bones vespres, sir.

(46) Et anut vous dirrez ainsi :

- Bon soer vous doint Dieux ou / - Sir, Dieu vous donne bon soer.

(47) Et quant vous prendrez congé de nully pur tout la nut, vous dirrez ainsi : I

- Sir, Dieu vous doint bon nut et bon repos, quar je m'en irray coucher.

À côté de formules qui présentent une organisation de la journée différente de l'actuelle, nous avons aussi des séquences disparues de l'usage. C'est le cas, par exemple, d'une formule de politesse fortement ritualisée qui concernait le moment où deux personnes s'apprêtaient à boire à la même table. Le pragmatème utilisé dans ces cas était : Prennez vostre hannap et commencés, qui était suivi de répliques comme Non ferrey devant vous et de contre-répliques (Vous ferez). Nous sommes devant un échange de civilités semblable à celui qui, de nos jours, peut se produire lors du franchissement d'une entrée : Après vous...

(48) - Dame, prennez vostre hanap et comencés.

- Mon seignour, s'il vous pleast, non fray devant vous.

- Par Dieu, si frez.

- Vostre merci, mon seignour. (ML 1399, p. 16)

Et, dans la Manière de langage de 1415 (Frantex 7010) : 
(49) - Sire, prennez le hanape, vous comencerez.

- Dame, non ferrey devant vous.

- Sire, vous ferez vrayement.

- Par sainte Marie, c'est bon boire. (p. 57)

Notons que l'acceptation de la préséance est accompagnée d'une dernière formule, soit pour exprimer le remerciement, soit pour exprimer le plaisir de boire. L'échange peut être clos encore par une autre formule (- Grant proue le vous face ou - Grant bien vous face il), ce qui nous donne un total de jusqu'à six types de formules pour une situation donnée.

\section{Conclusions}

Nous avons essayé de montrer comment une méthodologie d'analyse linguistique formelle peut être appliquée à la description du français médiéval (ancien français et moyen français) et à la problématique de la traduction entre celui-ci et la langue de nos jours. Bien entendu, nous n'avons pu présenter que quelques exemples. Cependant, étant donné qu'elle possède une adéquation descriptive et des capacités explicatives, cette méthodologie, à l'origine mise au point principalement pour des applications en linguistique informatique, peut être mise à profit par tout un éventail de domaines visant des applications fort diverses. Il s'agit, par conséquent, d'un outil polyvalent qui, situé à la base de la formation de l'enseignant/chercheur, peut lui permettre de mener à bien sa tâche de manière précise, cumulable et reproductible, c'est-à-dire, de façon scientifique. Le fait que toute discipline soit basée, d'une façon ou d'une autre, sur un corpus textuel qui relève d'une utilisation particulière de la langue, fait de la linguistique un outil transversal.

Mais nous nous tromperions également si nous ne contemplions la linguistique (en particulier, la linguistique française) que comme un mécanisme d'analyse et de transformation de textes et si nous envisagions son enseignement uniquement dès une perspective purement technique. Comme l'a fait très justement remarquer Julio Murillo dans sa présentation du premier numéro de la revue Langue $(s)$ \& Parole (UAB-CIPA) :

[...] l'analyse d'une langue, le français en l'occurrence, et a fortiori son enseignementapprentissage, va bien au-delà de l'acte didactique ou de l'analyse linguistique stricto sensu. Il s'ensuit que la fonction même et le rôle d'un Département universitaire de français et d'un organisme comme le Centre International de Phonétique Appliquée ne se circonscrit pas à enseigner et analyser les langues et la parole comme s'il s'agis- 
sait de maîtriser un nouvel instrument qui permet à un citoyen du $\mathrm{XXI}^{\mathrm{e}}$ siècle d'élargir ses propres relations et de communiquer avec des personnes qui parlent une autre langue que la sienne, mais également, et peut-être surtout, de définir, de préciser et de transmettre des valeurs.

[...] Au cours des dernières décennies, en particulier comme réaction à des conceptions totalitaires, dogmatiques et unitaristes de l'individu et de la société, il n'est pas exceptionnel qu'une lecture biaisée et réductrice des principes de l'humanisme, du libre-arbitre et des fondements des droits et libertés ait parfois débouché sur une sorte de relativisme généralisé sans points de repère, et qui s'avère impuissant pour s'opposer au fanatisme et à l'intolérance.

L'analyse et la diffusion des langues, des concepts qu'elles expriment et véhiculent, acquièrent dès lors leur pleine dimension sociétale et constituent des actions appelées à jouer un rôle capital dans la formation du citoyen. Par le truchement des recherches sur la parole (aux plans phonique, lexico-sémantique, morphosyntaxique ou textuel) et par l'enseignement-apprentissage des langues et des cultures qu'elles expriment et véhiculent, c'est la « formation des esprits » qui est en jeu, qui est l'enjeu, et dont la portée ne peut être sous-estimée.

$(2015: 9-11)$

À notre avis, il faut d'autant plus tenir compte de ces propos que des déficits dans l'enseignement des humanités peuvent entraîner une fâcheuse tendance à des approches basées sur un nemo ante me qui n'aurait plus rien de cartésien, mais qui relèverait plutôt d'une ignorance devenue militante. En face de ces dérives, la réponse est de conserver, développer et diffuser des savoirs et des savoir-faire ancrés dans la meilleure tradition européenne, tout en gardant à l'esprit que la bonne façon de préserver une tradition consiste à la débarrasser du superflu pour en garder l'essentiel. Tel a été, nous semble-t-il, le défi relevé avec succès par Gaston Gross et sa méthodologie d'analyse linguistique.

\section{Références citées}

Blanco, X. (2015). Pragmatèmes français du XIV siècle dans les Manières de langage. In P. Mogorrón \& F. Navarro (Éds), Fraseología, didáctica y traducción (p. 3-66). Frankfurt a.M., Peter Lang.

Blanco, X. (2018). La traduction des verbes supports de l'ancien français. Le Français moderne, 86(1), 3-54.

Blanco, X. (2020). Remarques sur la variation diachronique des collocations. Cahiers de Lexicologie, 116, 71-94.

Blanco, X. (sous presse). Le sang, le feu et la rose. La couleur rouge comme tertium comparationis en français médiéval. In G. Gross, F. Neveu \& M. Fasciolo, (Éds), Décrire une langue : objectifs et méthodes. Paris, Classiques Garnier. 
Blanco, X., \& Mejri, S. (2018). Les pragmatèmes. Paris, Classiques Garnier.

Dostie, G. (2019). Paramètres pour définir et classer les phrases préfabriquées : « La vengeance est un plat qui se mange froid. Bon appétit! ». Cahiers de Lexicologie, 114, 27-61.

García Pérez, R. (2005). ¿Desde cuándo se cometen delitos? Relaciones entre léxico y sintaxis en la evolución histórica de la lengua del Derecho penal. In L. Santos Río et al. (Eds), Palabras, norma, discurso. En memoria de Fernando Lázaro Carreter (p. 509-519). Salamanca, Universidad de Salamanca.

Gross, G. (2012). Manuel d'analyse linguistique. Villeneuve-d'Ascq, Presses universitaires du Septentrion.

Le Pesant, D., \& Mathieu-Colas, M. (1998). Introduction aux classes d'objets. Langages, $131,6-33$.

Mel'čuk, I. (2020). Clichés and pragmatemes. Neophilologica, 32, 9-20.

Mel'čuk, I., \& Polguère, A. (sous presse). Les fonctions lexicales dernier cri. In S. Marengo (Éd.), La Théorie Sens-Texte et ses applications. Lexicologie, lexicographie, terminologie, didactique des langues. Paris, L'Harmattan.

Murillo, J. (2015). Présentation. Langue(s) \& Parole, 1, 9-14.

Pustejovsky, J. (2016). The Generative Lexicon. Cambridge (Massachusetts), MIT Press.

Rolland-Perrin, M. (2010). Blonde comme l'or. La chevelure féminine au Moyen Âge. Senefiance, 57. Aix-en-Provence, Presses universitaires de Provence.

\section{Sources primaires}

1797. Théâtre de P. Corneille avec les commentaires de Voltaire (T. 7 et T. 8). Paris, Bossange, Masson et Besson.

Clari, R. de (2004). La conquête de Constantinople. J. Dufournet (Éd.). Paris, Honoré Champion.

Harf-Lancner, L. (Éd.). (1994). Le Roman d'Alexandre. Paris, Librairie Générale Française, Le livre de poche, coll. « Lettres gothiques ».

Henry, A. (Éd.). (1982). Adenet le Roi. Berte as grans piés. Genève, Droz.

Joly, A. (Éd.). (1870-71). Le Roman de Troie ou les métamorphoses d'Homère et de l'épopée gréco-latine au Moyen Âge (T. 2). Paris, Franck.

Laurent, F., Le Saux, F., \& Bragantini-Maillard, N. (Éds). (2019). Vie de sainte Marguerite. Conception Nostre Dame. Vie de Saint Nicolas. Paris, Honoré Champion.

Lepage, Y. G., \& Ollier, M.-L. (Éds). (2002). Le Val des amants infidèles (Vol. IV : Lancelot $d u$ Lac). Paris, Librairie Générale Française, Le livre de poche, coll. « Lettres gothiques ».

Martin, J.-P. (Éd.). (2014). Beuve de Hamptone. Paris, Honoré Champion.

Méla, Ch., \& Blons-Pierre, C. (Éds). (1997). Chrétien de Troyes, Perceval ou le Conte du Graal. Paris, Librairie générale française.

Potvin, Ch. (Éd.). (1866-71). Perceval le Gallois ou le Conte du Graal (T. 6). Mons, Dequesne-Masquillier. 
Roach, W., \& Ivy, R. H. (Eds.). (1949-50). The Continuations of the Old French "Perceval" of Chrétien de Troyes (Vol. 2: The First Continuation). Philadelphia, University of Pennsylvania Press.

Thiry, Cl. (Éd.). (1991). Villon. Poésies complètes. Paris, Librairie Générale Française, Le livre de poche, coll. « Lettres gothiques ».

Villehardouin, G. de (1972). La conquête de Constantinople (2 vols). E. Faral (Éd.). Paris, Les Belles Lettres.

Weiss, J. (Éd.). (2008). Boeve de Haumtone and Gui de Warewic. Tempe, Arizona Center for Medieval and Renaissance Studies.

Wienbeck, E., \& Rasch, P. (Éds). (1903). Aliscans. Halle a. S., Niemeyer.

\section{Bases textuelles et dictionnaires}

AND : The Anglo-Normand Dictionary, AND2 Online edition. https://anglo-norman.net/ (consulté le 10.06.2021).

BFM : Base de Français Médiéval. Lyon : ENS de Lyon, Laboratoire IHRIM, 2019. http:// bfm.ens-lyon.fr/ (consulté le 15.06.2021).

DMF : Dictionnaire du Moyen Français (1330-1500), ATILF — CNRS \& Université de Lorraine http://www.atilf.fr/dmf (consulté le 16.06.2021).

FRANTEXT : Frantext, ATILF — CNRS \& Université de Lorraine. https://www.frantext. fr (consulté le 16.06.2021).

GD : Dictionnaire de l'ancienne langue française et de tous ses dialectes $d u I X^{e}$ au XV siècle, par Frédérick Godefroy (1881). http://micmap.org/dicfro/search/dictionnairegodefroy (consulté le 15.06.2021). 